\title{
A Proof of the Crossing Property for Two-Particle Amplitudes in General Quantum Field Theory
}

\author{
By \\ J. BROS \\ C.E.N. Saclay, Gif-sur-Yvette (France) \\ H. EPSTEIN* \\ Institut des Hautes Etudes Scientifiques \\ Bures-sur-Yvette (France) \\ and T. GLASER \\ C.E.R.N., Geneva (Switzerland)
}

\begin{abstract}
In the framework of the $\mathscr{L} . \mathscr{S} . \mathscr{Z}$.formalism, the crossing property is proved on the mass shell for amplitudes involving two incoming and two outgoing stable particles with arbitrary masses. Any couple of physical regions in the $(s, t, u)$ plane corresponding to crossed processes are shown to be connected by a certain domain of analyticity. For every negative value of $t$, the amplitude is analytic in the cut $s$-plane outside of a large circle.
\end{abstract}

\section{Introduction}

In this paper we propose to prove the property of "crossing" for the scattering amplitudes involving four stable particles $A_{j}(j=0,1,2,3)$ with arbitrary non-zero masses $m_{j}$, within the framework of the $\mathscr{L} . \mathscr{S} . \mathscr{Z}$. formalism [1].

In this formalism the amplitudes corresponding to the processes

$$
\begin{array}{ll}
A_{1}+A_{2} \rightarrow A_{3}+A_{0} & \text { (and C.T.P.) } \\
A_{1}+A_{3} \rightarrow \bar{A}_{2}+A_{0} & \text { (and C.T.P.) } \\
\bar{A}_{3}+A_{2} \rightarrow A_{1}+A_{0} & \text { (and C.T.P.) }
\end{array}
$$

are differentboundary values of a holomorphic function $H(k)$ of the set of complex four-vectors

$$
k=\left\{k_{0}, k_{1}, k_{2}, k_{3} \underset{j=0}{\Sigma} k_{j}=0\right\} \quad H(k) \text { is defined }
$$

and analytic in a certain primitive domain ${ }^{\star \star} \Delta$ in $\mathbb{C}^{12}$. More precisely if $\boldsymbol{P}_{j}$ is the four-momentum of the particle $A_{j}\left(\operatorname{or} \bar{A}_{j}\right)\left(P_{j} V^{+}\right.$, for $\left.j=0,1,2,3\right)$ and

* C.N.R.S., France.

** For a description of this domain and for relevant references, we refer the reader to a previous paper [2], the notations of which will also be used in the present paper. 
if $T^{(i)}\left(P_{j}\right)$ denotes the amplitude of the reaction $(i)(i=1,2,3)$, then we have:

$$
T^{(i)}\left(P_{j}\right)=\lim H(k), k_{j} \rightarrow \varepsilon_{i j} P_{j} .
$$

In these formulae, the limit has to be taken in the sense of distributions for $k$ lying in certain tubes contained in $\Delta$ the number $\varepsilon_{i j}$ is equal either to +1 or to -1 according to whether the particle $A_{j}$ (or $\bar{A}_{j}$ ) is incoming or outgoing in the reaction (i).

Actually, it tarns out that the section of the primitive domain $\Delta$ by the "mass shell" manifold $\left\{k ; k_{j}^{2}=m_{j}^{2} 0 \leqq j \leqq 3\right\}$ is empty.

The purpose of this work is to prove that the envelope of holomorphy $\mathscr{H}(\Delta)$ of $A$ always contains a connected open set of the mass shell manifold which connects the physical regions of the reactions (1), (2), (3); this is what we mean by "crossing." Of course this property has to be supplemented by the fact that the formula (4) still holds true in the sense of distributions when we replace $H(k)$ by the well-defined analytic function which is the restriction to the mass shell of the analytic continuation of $H(k)$ however we postpone the proof of this point to a further paper and are only concerned here with the questions of analyticity domains. A typical consequence of the property of crossing is that the knowledge of one of the amplitudes $T^{(i)}\left(P_{j}\right)$ should in principle be sufficient to determine uniquely the amplitudes of the crossed reactions.

In order to describe our domain of crossing, we introduce the usual Lorentz invariant coordinates :

$$
\begin{gathered}
s=\left(k_{1}+k_{2}\right)^{2} ; \quad t=\left(k_{1}+k_{3}\right)^{2} ; \quad u=\left(k_{2}+k_{3}\right)^{2} \\
\zeta_{j}=k f \quad(j=0,1,2,3) .
\end{gathered}
$$

These variables are related by the formula:

$$
*+t+u=\sum_{j=0}^{8} \zeta_{j} \text {. }
$$

Let us denote $I$ the mapping $k \rightarrow\left(s, t, u, \zeta_{j}\right)$, and put:

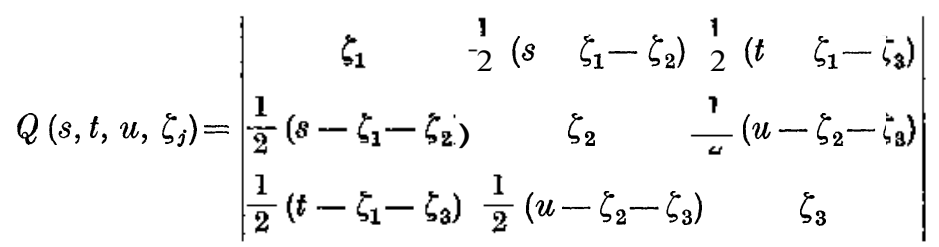

(defined on the manifold (5)). $Q(I(k))$ is the Gramian of $\left(k_{1}, k_{2}, k_{3}\right)$ (or of $\left(k_{1}, k_{2}, k_{0}\right)$, etc.).

When $\zeta_{j}=m f(j=0,1,2,3)$, there are three real regions in the space of the variables $s, t, u$ (the Mandelstam-plane), denoted $S, T, U$, corresponding to all possible values of $s, t, u$ in the processes (1), (2), (3) respectively (or their C.T.P. transforms). As it is well known, these 
regions are bounded by branches of the cubic $\left\{Q\left(s, t, u, m_{j}^{2}\right)=0\right\}$ (see Fig. 1).

We shall prove that $\mathscr{H}(A)$ contains an open connected subset of the mass shell which has the following properties:

a) This domain is invariant under complex Lorentz transformations and can be defined by

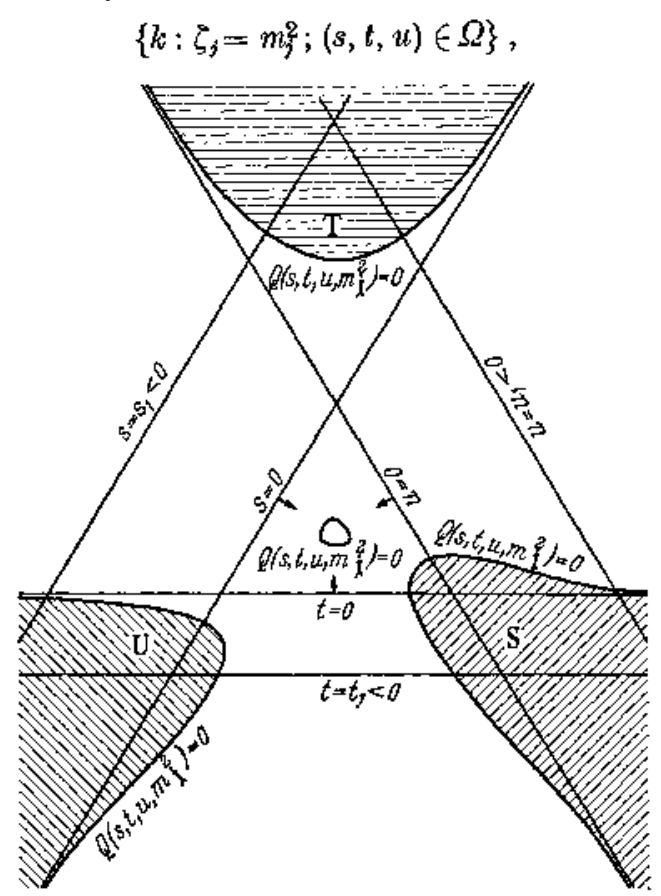

Fig. 1. The physical regions $S, T, U$ in the $(s, t, u)$ - plane

where $\Omega$ is a connected open set (domain) in the space of the variables $(\mathrm{s}, t, u)$ related by:

$$
s+t+u=\Sigma m_{j}^{2} .
$$

b) The open set $\Omega$ contains six disjoint subdomains $S^{ \pm}, T^{ \pm}, U^{ \pm}$of the following form:

$$
S^{ \pm}=r f(8) \cap\{(s, t, u): \pm \operatorname{Im} s>0 ; Q(s, t u, m f) \neq 0\}
$$

where $\mathscr{N}(8)$ is a certain open connected neighbourhood of $S$ (on the mass shell). $T^{ \pm}$and $U^{ \pm}$have similar forms.

When $k$ tends on the mass-shell to a point of $S, T, U$ from $S^{+}, T^{+}, U^{+}$ respectively, the restriction of $H(k)$ to the mass-shell will tend to the physical amplitudes of the reactions (1), (2), (3) respectively. 
c) For any real negative value $t_{1}<0$, there exists an open subset $\Omega_{t}^{+}\left(t_{1}\right)$ (resp. $\left.\Omega_{t}^{-} f a\right)$ ) of $\Omega$ of the form:

$$
\left\{(\mathrm{s}, t, u) \quad \operatorname{Im} s>0(\text { resp. } \operatorname{Im} s<0) ;|\mathrm{s}|>R\left(t_{1}\right) ;\left|\hat{\imath}-t_{1}\right|<\varepsilon\left(t_{1}, s\right)\right\} .
$$

$\Omega_{t}^{+}\left(t_{1}\right)$ connects $S^{+}$and $U^{-} \Omega_{t}^{-}\left(t_{1}\right)$ connects $S^{-}$and $U^{+}$. Analogous domains $\Omega_{s}^{ \pm}\left(s_{1}\right)$ and $\Omega_{u}^{ \pm}\left(u_{1}\right)$ can be constructed by obvious permutations of the variables: $\Omega_{u}^{+}\left(u_{1}\right)$ connects $T^{+}$and $S^{-} \Omega_{n}^{-}\left(u_{1}\right)$ connects $T^{-}$and $S^{+}$, $\Omega_{s}^{+}\left(s_{1}\right)$ connects $U^{+}$and $T^{-}$and $\Omega_{s}^{-}\left(s_{1}\right)$ connects ' $O \sim$ and $T^{+}$. The announced domain $\Omega$ can be taken to be the union of $S^{ \pm}, T^{ \pm}, U^{ \pm}, \Omega_{s}^{ \pm}\left(s_{1}\right)$, $\Omega_{t}^{ \pm}\left(t_{1}\right), \Omega_{u}^{ \pm}\left(u_{1}\right)$ (for all negative $s_{1}, t_{1}, u_{1}$ ). This is clearly a connected set. One notices that, to pass from one physical region to another within $\Omega$, one must follow a path which approaches the third physical region from the "unphysical side": for instance a path going from $S^{+}$to $U^{+}$must intersect $T^{-}$.

The only assumptions needed on the masses of the theory are the usual stability conditions (see [2] §5), and the existence of a strictly positive minimum mass for all states other than the vacuum.

We first note the following facts which play an important role for the rigour of the proof.

1. The hypothesis that there is a minimum mass in the theory implies that $\Delta$ contains the origin 0 of the coordinates, and is star-shaped with respect to 0 . It can be shown that $\mathscr{H}(\Delta)$ is then necessarily a star-shaped domain in $\mathbb{C}^{12}$, i. e. it is schlicht. Therefore, if a point of $\mathscr{H}(A)$ has been obtained by two different methods, the corresponding continuations of $H(k)$ will nevertheless agree at that point.

2. $f f l(\Delta)$ is necessarily invariant under the complex Lorentz group $L_{+}(\mathbb{C})$. This follows from $\mathscr{H}(\Delta)$ being "schlicht," and from a process of analytic completion described in reference [3] and [4]. It is also invariant under reflections, because $A$ has this in variance. Hence: $\mathscr{H}(\Delta)$ is invariant under $L(\mathbb{C})$, the full complex Lorentz group.

3. The following property has been proved by HALL and WightMaN in [5]: let $k$ and $k^{\prime}$ be points having the same set of scalar products $k_{i} \quad k_{j}=k_{i}^{\prime} \quad k_{j}^{\prime}$, and having non-zero Gramian $Q(I(k))$.

Then there exists $\Lambda \in L(\mathbb{C})$ such that $\Lambda k^{\prime}=k$. It follows that once the manifold $\{Q(I(k))=0\}$ is removed from $\mathscr{H}(\Delta)$, on obtains a domain which is the whole inverse image of its image in the invariants: in the terminology of HEPP [6], If $(\Delta) \Pi\{Q(I(k)) \neq 0\}$ is an $I$-saturated domain.

The property a) of our domain will be a trivial consequence of these general facts, once we have checked that all the above enumerated sets the union of which constitute $\Omega$ avoid the manifold $\{Q(I(k))-0\}$.

The proof of the property $b$ ), namely the existence of the domains $S^{ \pm}, T^{ \pm}, U^{ \pm}$,can then be deduced from the fact, proved in [2], that $\mathscr{H}(\Delta)$ 
contains cut neighbourhoods of all physical real points. (It is to be noted that the latter result can be obtained without the use of the Steinmann identities. For instance, to obtain $S^{ \pm}$, it is sufficient to apply a local edge of the wedge theorem to the "tubes" $\left(\mathscr{T}_{01}^{+}+\mathscr{T}_{32}^{-}\right) \Pi C \Gamma_{12}$ and $\left(\mathscr{T}_{01}^{-}+\mathscr{T}_{32}^{+}\right)$ $\cap \mathrm{C} \Gamma_{12}$ The argument is exactly similar to that of [2], §5).

The remaining sections of the present paper are thus devoted to proving the property c). A heuristic sketch of the proof can be given as follows.

Two kinds of domains of analyticity invariant under $L(\mathbb{C})$ are constructed. Their images in the invariants are of the types $D^{(1)}$ and $D^{(2)}$ which will now be described.

The momentum transfer $t=\left(k_{1}+k_{3}\right)^{2}$ is fixed at a negative value and the variables $\zeta_{j}$ are restricted to satisfy:

$$
\zeta_{k}-\zeta_{j}=m_{k}^{2}-m_{j}^{2}(0 \leqq k \leqq j \leqq 3)
$$

the only remaining variables are then:

$$
\zeta=\sum_{0 \leqq i \leq 3} \zeta_{1}, \quad s=\left(k_{1}+k_{2}\right)^{2}, \quad u=\left(k_{2}+k_{3}\right)^{2}
$$

which satisfy (see (5)):

$$
\zeta-s-u-t \text {. }
$$

$D^{(\mathbf{d})}$ is an open neighbourhood of the topological product of a part $R^{(1)}$ of the negative real axis in the $\zeta$-plane, by the upper half-plane in the variable $s$ (see Fig. 2).
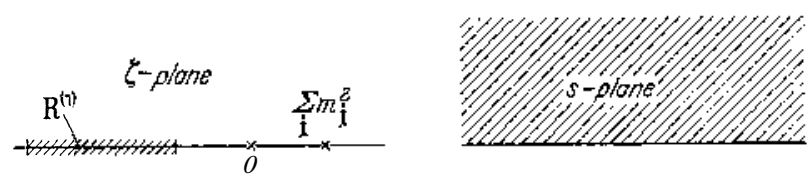

Fig. 2. Domain $D^{(1)}$ (shaded)

$D^{(2)}$ is an open neighbourhood of the topological product of a domain $R^{(2)}$ of the $\zeta$-plane by the union of two half-lines $S^{(2)}, U^{(2)}$ of the $s$-plane (parallel to the real $s$-axis) having the following properties (see Fig. 3 ):

$R^{(2)}$ contains $R^{(1)}$ and the "physical point"

with

$$
\begin{gathered}
\zeta=\sum_{j=0}^{8} m_{j}^{2} \\
S^{(2)}=\left\{s ; \operatorname{Im} s=\varepsilon>0 ; s-s_{1}>0\right\} \\
U^{(2)}=\left\{s ; \operatorname{Im} s=\varepsilon>0 ; s-s_{2}<0\right\}
\end{gathered}
$$

$$
\begin{gathered}
s_{2}-s_{1}<0 \operatorname{Im} s_{1}=\operatorname{Im} s_{2}=\varepsilon \\
S^{(2)} \subset S^{+} \quad U^{(2)} \subset U^{-} .
\end{gathered}
$$

An analytic completion of $D^{(1)} \cup D^{(2)}$ is then performed (see section 5) by using a certain application of the semi-tube theorem (local version). 
The intersection of this extended domain with the manifold

contains a domain in $s$ of the form:

$$
\left\{\zeta=\sum_{j} m_{j}^{2}\right\}
$$

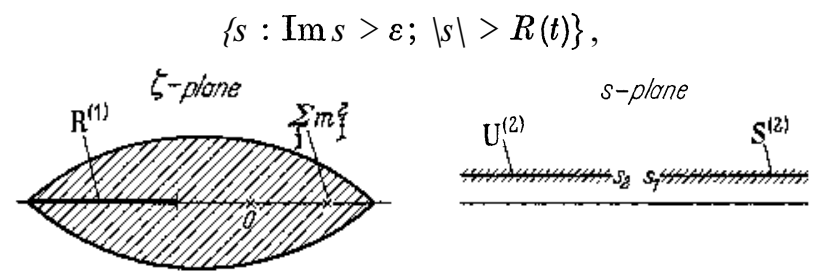

Fig. 3. Domain $D^{(2)}$ (shaded)

which yields a connection, on the mass-shell, between $S^{(2)}$ and $U^{(2)}$, and therefore between $S^{+}$and $U^{-}$.

It may be noticed that, apart from the method of analytic completion, the geometrical situation is somewhat similar to that of the classical proofs of dispersion relations $([7-11])$ in that it uses a domain of type $D^{(1)}$ and the complex variables $s$ and $\zeta$.

In order to obtain domains of type $D^{(1)}$ and $D^{(2)}$, we study the restriction of $H(k)$ to a linear submanifold $\mathscr{V}$. In section 2 , the manifold $\mathscr{V}$ will be defined and studied, and a glimpse of the construction of $D^{(1)}$ and $D^{(2)}$ will be given. In sections, $3,4,5$, the proof will be given in detail.

\section{The Manifold if}

The manifold if is defined uniquely up to a sign by the following conditions:

i) The fourth components $k_{j}^{(3)}$ of $k_{0}, k_{1}, k_{2}, k_{3}$ are taken to be zero, i. e. one considers only vectors of a 3 -dimensional space-time.

ii) $k_{1}+k_{3}=-\left(k_{2}+k_{0}\right)$ is held fi ${ }^{\text {xe }} \mathrm{i}$, real, along the third coordinate axis, with $\left(k_{1}+k_{3}\right)^{2}=t<0$.

iii) The third components of $k_{1}, k_{2}, k_{3}$ are held fixed and real.

iv) The manifold $\mathscr{V}$ must contain points on the mass-shell i. e. the system $k_{j}^{2}=m_{j}^{2}(0 \leqq j \leqq 3)$ must be compatible on $\mathscr{V}$.

The manifold $\mathscr{V}$ so defined may be parametrized by two complex vectors $\pi_{1}$ and $\pi_{2}$ of a two-dimensional space-time as follows:

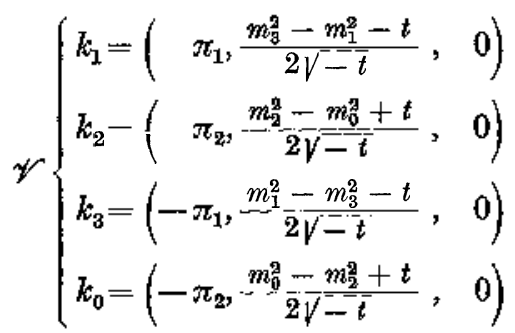


The vector $\pi_{j}-\left(\pi_{j}^{(0)} \pi_{j}^{(1)}\right)(j=1,2)$ will often be represented by isotropic coordinates:

$$
\left\{\begin{array}{l}
u_{j}=\pi_{j}^{(0)}+\pi_{j}^{(1)} \\
v_{j}=\pi_{j}^{(0)}-\pi_{j}^{(1)}
\end{array} \quad(j=1,2)\right.
$$

Their scalar products in the Lorentz metric can be expressed:

$$
\begin{gathered}
\pi_{j}^{2}=\left(\pi_{j}^{(0)}\right)^{2}-\left(\pi_{j}^{(1)}\right)^{2}=u_{j} v_{j} \\
\pi_{1} \cdot \pi_{2}=\pi_{1}^{(0)} \pi_{2}^{(0)}-\pi_{1}^{(1)} \pi_{2}^{(1)}=\frac{1}{2}\left(u_{1} v_{2}+u_{2} v_{1}\right) .
\end{gathered}
$$

We notice that in the manifold $\mathscr{V}$, two mass differences are held fixed, namely:

$$
\left\{\begin{array}{l}
\zeta_{1}-\zeta_{s}=r_{1}^{2}-L_{s}^{2}=m_{1}^{2}-m_{s}^{2} \\
\zeta_{0}-\zeta_{2}=k_{0}^{2}-k_{2}^{2}=m_{0}^{2}-m_{2}^{2}
\end{array}\right.
$$

By Theorem 4 of [2] the intersection of $\mathscr{H}(\Delta)$ with $\mathscr{V}$ contains the eight following tubes, which are "faces" of the tubes $\mathscr{T}_{j}^{ \pm}, \mathscr{T}_{j \mathfrak{j}}^{ \pm}$:

$$
\begin{aligned}
& \mathscr{A}=\left\{\left(\pi_{1}, \pi_{2}\right) \quad \operatorname{Im} \pi_{1} \in V^{-} \quad \operatorname{Im}\left(\pi_{1}+\pi_{2}\right) \in V^{+}\right\} \\
& \mathscr{A}^{\prime}=\left\{\left(\pi_{1}, \pi_{2}\right) \quad \operatorname{Im} \pi_{1} \in V^{+} \quad \operatorname{Im}\left(\pi_{1}-\pi_{2}\right) \in V^{\prime \prime}\right\} \\
& \mathscr{B}=\left\{\left(\pi_{1}, \pi_{2}\right) \quad \operatorname{Im} \pi_{2} \in V^{-} \quad \operatorname{Im}\left(\pi_{1}+\pi_{2}\right) \in V^{+}\right\} \\
& \mathscr{B}^{\prime}=\left\{\left(\pi_{1}, \pi_{2}\right) \quad \operatorname{Im} \pi_{2} \in V^{-} \quad \operatorname{Im}\left(\pi_{1}-\pi_{2}\right) \in V^{-}\right\}
\end{aligned}
$$

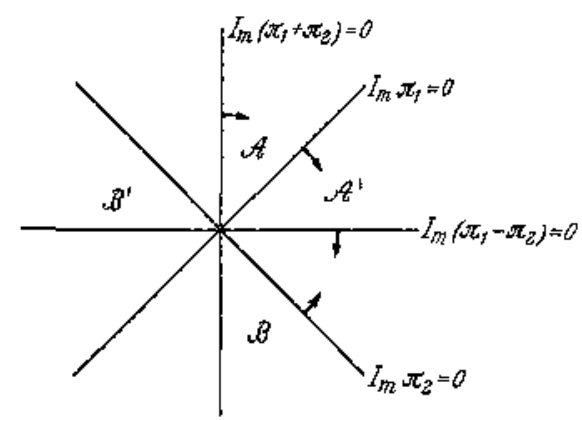

Fig. 4. Symbolic representation of the 8 tubes in $\mathscr{V}$ the arrows indicate the direction where the corresponding vectors lie in $V^{+}$

and their opposites (see Fig. 4). These tubes are mutually connected by certain open sets of $\operatorname{ffl}(A] \Pi \mathscr{V}$ near certain real points [2]. In particular $f f l(\Delta) \Pi \mathscr{V}$ contains open sets $\mathscr{N} \cap(\mathscr{A}+\mathscr{B})$ and $\mathscr{N}^{\prime} \cap\left(\mathscr{A}^{\prime}+\mathscr{B}^{\prime}\right)$ connecting $\mathscr{A}$ with $\mathscr{B}$ and $\mathscr{A}^{\prime}$ with $\mathscr{B}^{\prime}$, respectively, where $\mathscr{N}$ is an open complex neighbourhood (in $\mathscr{V}$ ) of the real points in $\mathscr{V}$ satisfying

$$
\pi_{1}^{2}<\mathscr{H}_{1}^{2}, \pi_{2}^{2}<\mathscr{M}_{2}^{2},\left(\pi_{1}-\pi_{2}\right)^{2}<\mathscr{M}_{\text {in }}^{2}
$$

and $\mathscr{N}^{\prime}$ is an open complex neighbourhood of the real points in $\mathscr{V}$ such that

$$
\pi_{1}^{2}<\mathscr{M}_{1}^{2}, \pi_{2}^{2}<\mathscr{M}_{2}^{2},\left(\pi_{1}+\pi_{2}\right)^{2}<\mathscr{M}_{12}^{2} .
$$


Here, one has defined:

$$
\begin{aligned}
& \mathscr{M}_{1}^{2}=\min \left\{M_{1}^{2}-\frac{1}{-}\left(t+m_{1}^{2}-m_{3}^{2}\right)^{2}, M_{3}^{2}-\frac{1}{4 t}\left(t+m_{3}^{2}-m_{1}^{2}\right)^{2}\right\} \\
& \left.\mathscr{M}_{2}^{2}=\min \left\{M_{2}^{2}-1-1+\mathrm{i}+m_{0}^{2}\right)^{2}, M_{0}^{2}-\frac{1}{4 t}\left(t+m_{0}^{2}-m_{2}^{2}\right)^{2}\right\} \\
& \mathscr{M}_{10}^{2}=M_{10}^{2}-\frac{1}{4 t}\left(m_{1}^{2}-m_{3}^{2}+m_{2}^{2}-m_{0}^{2}\right)^{2} \\
& \mathscr{M}_{12}^{2}=M_{12}^{2}-\frac{1}{4 t}\left(m_{1}^{2}-m_{3}^{2}-\mathbf{m i}+m_{0}^{2}\right)^{2} .
\end{aligned}
$$

$(\mathscr{A}+\mathscr{B})\left(\right.$ resp. $\left.\mathscr{A}^{\prime}+\mathscr{B}^{\prime}\right)$ denotes the convex hull of $\mathscr{A} \mathrm{U} \mathscr{B}$ (resp. $\left.\mathscr{A}^{\prime} \mathrm{U} \mathscr{B}^{\prime}\right)$ namely $\left\{\left(\pi_{1}, \pi_{2}\right) \operatorname{Im}\left(\pi_{1}+\pi_{2}\right) \in V^{+}\right\}\left(\operatorname{resp} .\left\{\left(\pi_{1}, \pi_{2}\right) \operatorname{Im}\left(\pi_{1}-\pi_{2}\right)\right.\right.$ $\left(\in V^{-}\right\}$). (It is easy to see that all these properties of $\mathscr{H}(\Delta) \Pi \mathscr{V}$ can be obtained without using the Steinmann identities).

The domains of the type of $D^{(1)}$ will be obtained by taking the image of certain of these tubes and their boundaries in the invariants. On the other hand those of the type of $D^{(2)}$ are given by images of partial analytic completions of the above eight-tube domain. In order to obtain them, we shall use the well-known Jost-Lehmann-Dyson domains [12, 13].

The intersection of $\mathscr{V}$ with the mass shell is contained in the submanifold $\mathscr{W}$ of $\mathscr{V}$ defined by

or alternatively, by:

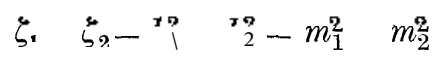

$$
\mathscr{W}^{\prime}=\left\{\left\{\left(\pi_{1}, \pi_{2}^{\prime}\right) ; \pi_{1}^{2}-\pi_{2}^{2}-\mu_{1}^{2}-\mu_{2}^{2}\right\}\right.
$$

where

$$
\begin{aligned}
& \mu_{1}^{2}=m_{1}^{2}-\frac{2}{A t}\left(t+m_{1}^{2}-m_{3}^{2}\right)^{2}=m_{3}^{2}-\frac{1}{4 t}\left(t+m_{3}^{2}-m_{1}^{2}\right)^{2} \\
& \mu_{2}^{2}=m_{2}^{2}-\frac{1}{4 t}\left(t+m_{2}^{2}-m_{0}^{2}\right)^{2}=m_{0}^{2}-\frac{1}{4 t}\left(t+m_{0}^{2}-m_{2}^{2}\right)^{2}
\end{aligned}
$$

We recall the following general fact, which is essential for the rigor of our proof: let $A$ be a domain and $M$ a (regular) complex submanifold of $\mathbb{C}^{n}$. Then the envelope of holomorphy of $A \Pi M$ (in $M$ ) is contained in the envelope of holomorphy of $A$ (see [14]). This shows, in particular, that every point $K=\left(K_{1}, K_{2}, K_{3}\right)$ which we shall obtain by analytic completion in $\mathscr{V}$ is an interior point of $\mathscr{H}(\Delta)$, i. e. $H(k)$ can be analytically continued in some neighbourhood

$$
\left\{k:\left\|k_{1}-K_{1}\right\|+\left\|k_{2}-K_{2}\right\|+\left\|k_{3}-K_{3}\right\|<\varepsilon\right\}
$$

of $K$. This can also be understood by remarking that our methods of completion can always be, in principle, reduced to the "disk theorem." 


\section{Passage to the invariants in $\mathscr{V}$}

The two following systems of variables will be used in the manifold $\mathscr{V}$.

(I) $u_{1}, v_{1}, u_{2} v_{2}$, or: $u^{+}, v^{+}, u^{-}, v^{-}$, where

and

$$
\left\{\begin{array}{l}
u^{ \pm 4}=u_{1} \pm u_{2} \\
v^{ \pm}=v_{1} \pm v_{2}
\end{array}\right.
$$

(II)

$$
\left\{\begin{array}{l}
z_{1}=\pi_{1}^{2}=u_{1} v_{1} \\
z_{2}=\pi_{2}^{2}=u_{2} v_{2} \\
z_{3}=\left(\pi_{1}+\pi_{2}\right)^{2}=\left(u_{1}+u_{2}\right)\left(v_{1}+v_{2}\right) \\
\alpha=\sqrt{\frac{u_{1}}{v_{1}}} .
\end{array}\right.
$$

The system of variables (II) corresponds to choosing three independent Lorentz invariants, $z_{1}, z_{2}, z_{3}$, and a variable $\alpha$ representing a complex Lorentz transformation in two-dimensional space-time. To study the regularity of this change of variables we write down the inverse formulae:

$$
\begin{aligned}
& u_{1}=\sqrt{z_{1}} \alpha, v_{1}=\frac{\sqrt{z_{1}}}{\alpha} \\
& u_{2}=\frac{\alpha}{2 \sqrt{z_{1}}}=-\left[z_{3}-z_{1}-z_{2}+\sqrt{\lambda\left(z_{3}, z_{1}, z_{2}\right)}\right] \\
& \left.v_{2}-\frac{1}{2 \alpha \sqrt{z_{1}}}-\quad z_{1} \quad z_{2} \quad, \quad\left(z_{3}, z_{1}, \overline{z_{2}}\right)\right]
\end{aligned}
$$

where $\lambda\left(z_{3}, z_{1}, z_{2}\right)$ is the symmetric form

$$
\begin{aligned}
& \lambda\left(z_{3}, z_{1}, z_{2}\right)=\left(z_{3}-z_{1}-z_{2}\right)^{2}-4 z_{1} z_{2}= \\
& \quad=z_{3}^{2}-2\left(z_{1}+z_{2}\right) z_{3}+\left(z_{1}-z_{2}\right)^{2}=\left(u_{1} v_{2}-u_{2} v_{1}\right)^{2} .
\end{aligned}
$$

The above formulae (21) imply: $\sqrt{\lambda}\left(z_{3}, z_{1}, z_{2}\right)=u_{2} v_{1}-u_{1} v_{2}$. Note that, on $\mathscr{V}: Q\left(s, t, u, \zeta_{j}\right)=-t \lambda\left(z_{1}, z_{2}, z_{3}\right)$. Two manifolds of critical points appear in the formulae; namely

and

$$
\lambda\left(z_{1}, z_{2}, z_{3}\right)=\mathbf{0}
$$

$$
z_{1}=0
$$

In order to avoid the critical points of the submanifold $z_{1}=0$, we introduce in $\mathscr{V}$ the cut:

$$
\begin{gathered}
\mathscr{C}=\left\{\left(u_{1}, v_{1}, u_{2}, v_{2}\right) \quad u_{1} v_{1} \notin \mathbb{R}^{-}\right\} \\
\left(\mathbb{R}^{-}=\text {set of all real numbers } \leqq 0\right) .
\end{gathered}
$$

The only domains of $\mathscr{V}$ of which we shall use the images in the space of the variables (II) will have to be contained in

$$
\mathscr{V}=\mathscr{V}-\mathscr{C} .
$$


The manifold $\lambda\left(z_{1}, z_{2}, z_{3}\right)=0$ is a manifold of ramification which can be suppressed as follows. The domain $\mathscr{H}(\Delta) \Pi \mathscr{V}^{\prime}$ is invariant under the Lorentz transformations:

$$
u_{j} \rightarrow \alpha u_{j}, v_{j} \rightarrow \frac{1}{\omega} v_{j}(j=1,2), \alpha \in \mathrm{C}-\{0\}
$$

and under the reflection $u_{j} \leftrightarrow v_{j}(j=1,2)$. Let $X$ be a subdomain of $\mathscr{H}(\Delta) \cap \mathscr{V}^{\prime}$. The union $X^{\prime}$ of the transforms of $X$ under all transformations of the above type is again in $\mathscr{H}(\Delta) \cap \mathscr{V}^{\prime}$ In particular $X^{\prime}$ is invariant under the following transformation (which is non-singular on $\left.\mathscr{V}^{\prime}\right)$ :

$$
\begin{aligned}
& u_{1} \rightarrow \frac{u_{1}}{v_{1}} v_{1}=u_{1}, v_{1} \rightarrow \frac{v_{1}}{u_{1}} u_{1}=v_{1} \\
& u_{2} \rightarrow \frac{u_{1}}{v_{1}} v_{2} \quad, v_{2} \rightarrow \frac{v_{1}}{u_{1}} u_{2} .
\end{aligned}
$$

The restriction of the 4-point function to $X^{\prime}$,

$$
H\left(k_{1}, k_{2}, k_{3}\right) / X^{\prime}=F\left(u_{1}, v_{1}, u_{2}, v_{2}\right)
$$

can then be written

$$
F\left(u_{1}, v_{1}, u_{2}, v_{2}\right)=F_{1}\left(u_{1}, v_{1}, u_{2}, v_{2}\right)+\left(u_{2} v_{1}-u_{1} v_{2}\right) F_{2}\left(u_{1}, v_{1}, u_{2}, v_{2}\right)
$$

where:

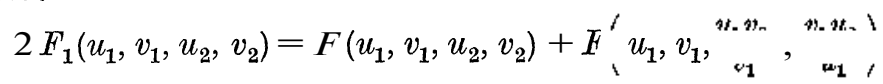

$$
\begin{aligned}
& 2 F_{2}\left(u_{1}, v_{1}, u_{2}, v_{2}\right)=\frac{F\left(u_{1}, v_{1}, u_{2}, v_{2}\right)-F\left(u_{1}, v_{1}, \frac{u_{1} v_{2}}{v_{1}}, \frac{v_{1} u_{2}}{u_{1}}\right)}{u_{2} v_{1}-u_{1} v_{2}}
\end{aligned}
$$

$F_{1}$ and $F_{2}$ are analytic in the same domain as $F$ and one can write:

$$
F_{1,2}\left(u_{1}, u_{2}, v_{1}, v_{2}\right)=f_{1,2}\left(z_{1}, z_{2}, z_{3}, \alpha\right)
$$

where $f_{1}$ and $f_{2}$ are holomorphic functions of the variables (II) having no singularities on the manifold $\lambda\left(z_{1}, z_{2}, z_{3}\right)=0$. Their domain of analyticity is the whole image of $X^{\prime}$ in the space of the variables (II). The computation of the envelope of holomorphy of $X^{\prime}$ is therefore equivalent to the computation of the envelope of holomorphy of its image in the variables (II).

We also define

$$
\mathscr{W}^{\prime}=\mathscr{W}-\mathscr{C}=\mathscr{V}^{\prime} \cap\left\{\left(u_{1}, v_{1}, u_{2}, v_{2}\right): u_{1} v_{1}-u_{2} v_{2}=\mu_{1}^{2}-\mu_{2}^{2}\right\}
$$

In $\mathscr{W}^{\prime}$, we shall use, for later analytic completions, the invariant variables $s, u$, and $z$, where $z$ is defined by the formula

where

$$
z=\sum_{j=0}^{3}\left(\zeta_{j}-m_{j}^{2}\right)-\Phi
$$

$$
\Phi=4 \min _{U \leq \backslash \leq}\left(M_{i}^{2}-m_{i}^{2}\right)-4 \min \left(\mathscr{M}_{1}^{2}-\mu_{1}^{2}, \mathscr{M}_{2}^{2}-\mu_{2}^{2}\right) .
$$


[The last equality follows from (12), (13), (17), (18)]; according to (5) $s, u$, and $z$ are related by:

$$
s+u+t=z+\Sigma m_{j}^{2}+\Phi .
$$

By virtue of the formulae (6), (17), (18), (20), $s, u$, and $z$ are related to the invariants $z_{1}, z_{2}$, and $z_{3}$ by the formulae:

$$
\begin{aligned}
& \left\{\begin{array}{l}
s=z_{3}+\frac{\left(m_{3}^{2}-m_{1}^{2}+m_{0}^{2}-m_{2}^{2}\right)^{2}}{4 t} \\
u=2\left(z_{1}+z_{2}\right)-{ }_{{ }_{3}}, \quad\left(m l-m l+m_{2}^{2}-m_{0}^{2}\right)^{2} .
\end{array}\right. \\
& z=2\left(z_{1}-\mu_{1}^{2}\right)+2\left(z_{2}-\mu_{2}^{2}\right)-\Phi .
\end{aligned}
$$

Due to the equation (16), which defines $\mathscr{W}$ we can also write:

$$
z=4\left(z_{1}-\mu_{1}^{2}\right)-\Phi=4\left(z_{2}-\mu_{2}^{2}\right)-\Phi \text { on } \mathscr{W}
$$

\section{Domains of analyticity in $\mathscr{V}$ and their images in the invariants}

Certain domains which will be described in this section can be used only for $\mu_{1}^{2}>\mu_{2}^{2}$. According to (17) and (18), this amounts to the following restriction on the momentum transfer $t$ :

$$
\left(m_{0}^{2}+m_{2}^{2}-m_{1}^{2}-m_{3}^{2}\right) t \geqq \frac{\left(m_{2}^{2}-m_{0}^{2}\right)^{2}-\left(m_{3}^{2}-m_{1}^{2}\right)^{2}}{2} .
$$

When the opposite inequality holds, one must use analogous domains obtained by exchanging the roles of $\pi_{1}$ and $\pi_{2}$.

\section{a) Domain $G_{1}$}

It has been shown in reference [2] (Theorem 4) that all points of if belonging to the boundaries of the eight tubes, described in section 2 , are in $\mathscr{H}(\Delta)$, provided they do not belong to one of the cuts:

$$
\begin{aligned}
& \left\{\left(u_{1}, v_{1}, u_{2}, v_{2}\right): u_{1} v_{1}=\mathscr{M}_{1}^{2}+\varrho_{1}, \varrho_{1} \geqq 0\right\} \\
& \left\{\left(u_{1}, v_{1}, u_{2}, v_{2}\right): u_{2} v_{2}=\mathscr{M}_{2}^{2}+\varrho_{2}, \varrho_{2} \geqq 0\right\} \\
& \left\{\left(u_{1}, v_{1}, u_{2}, v_{2}\right):\left(u_{1}+u_{2}\right)\left(v_{1}+v_{2}\right)=\mathscr{M}_{12}^{2}+\varrho_{3} \varrho_{3} \geqq 0\right\} \\
& \left\{\left(u_{1}, v_{1}, u_{2}, v_{2}\right):\left(u_{1}-u_{2}\right)\left(v_{1}-v_{2}\right)=\mathscr{M}_{01}^{2}+\varrho_{4}, \varrho_{4} \geqq 0\right\}
\end{aligned}
$$

(Here again, it is easy to show that this result can be obtained without using the Steinmann identities.) In particular the following sets of points belonging to the boundary of $\mathscr{A}$ (and of $\mathscr{A}^{\prime}$ ) lie inside $\mathscr{H}(\Delta)$ :
i) $u_{1}=v_{1}$ real $>0 ; u_{1}^{2}<\mathscr{M}_{1}^{2} ; \operatorname{Im} u_{2} \geqq 0 ; \operatorname{Im} v_{2}>0$.
ii) $v_{1}=0 ; u_{1}$ real $>0 ; \operatorname{Im} u_{2} \geqq 0$; $\operatorname{Im} v_{2}>0$.
iii) $\%=-v_{1}$ real $>0 \operatorname{Im} u_{2} \geq 0 \operatorname{Im} v_{2}>0$. 
Let $\mathscr{J}$ denote the continuous mapping which associates to every point $\left(u_{1}, v_{1}, u_{2}, v_{2}\right)$ of $\mathscr{V}$ the point $\left(z_{1}=\% v_{1}, z_{2}=u_{2} v_{2}, z_{3}=\left(u_{1}+u_{2}\right)\left(v_{1}+v_{2}\right)\right)$ of $\mathbb{C}^{3}$. We shall now verify that the image under $\mathscr{J}$ of the points just described contains the following set:

$$
E=\left\{\left(z_{1}, z_{2}, z_{3}\right): z_{1} \text { real }<\mathscr{M}_{1}^{2} z_{2} \text { real } \leqq 0 \operatorname{Im} z_{3}>0\right\}
$$

(i) $0<z_{1}<\mathscr{M}_{1}^{2} \quad u_{1}-v_{1}=\sqrt{z_{1}}$ if one fixes $z_{2}=u_{2} v_{2}$ real $<0$, the conditions $\operatorname{Im} u_{2}>0$ and $\operatorname{Im} v_{2}>0$ become equivalent one then has:

$$
z_{3}=z_{1}+z_{2}+\sqrt{z_{1}}\left(v_{2}+\frac{z_{2}}{v_{2}}\right),
$$

and it is clear that $z_{3}$ varies over the whole of $\left\{\operatorname{Im} z_{3}>0\right\}$ when $v_{2}$ varies over $\left\{\operatorname{Im} v_{2}>0\right\}$.

(ii) $z_{1}=0 \quad u_{1}$ real $>0 \quad v_{1}=0$ one fixes $z_{2}=u_{2} v_{2}$ real $<0$. The variable $z_{3}=z_{2}+u_{1} v_{2}$ varies over $\left\{\operatorname{Im} z_{3}>0\right\}$ when $v_{2}$ varies over $\left\{\operatorname{Im} v_{2}>0\right\}$.

(iii) $z_{1}<0 ; u_{1}=-v_{1}=V-z_{1}$, one fixes $z_{2}=u_{2} v_{2}$ real $<0$; then $z_{3}=z_{1}+z_{2}+V-z_{1}\left(v_{2} \frac{-}{v_{i}}-1\right.$ when $v_{2}$ varies over $\left\{\operatorname{Im} v_{2}>0\right\}, \mathrm{z}_{3}$ varies over a whole cut plane, in particular it takes all values in the upper half-plane.

In the three cases, the limiting case $z_{2}=0$ can be obtained by setting $u_{2}=0$. Then $z_{3}=z_{1}+\% v_{2}$; hence $z_{3}$ again takes all values in the upper half-plane when $v_{2}$ varies in $\left\{\operatorname{Im} v_{2}>0\right\}$.

We now come to a rather fine point in the argument, regarding the passage to the variables (II). The reader will notice that certain analyticity points described above ((ii) and (iii)) belong to the cut $\mathscr{C}$ (formula (23)) which should be avoided. But, in effect, since these points are in $\mathscr{H}(\Delta)$, which is open and Lorentz invariant, we know that there exists a certain open neighborhood $\mathscr{G}_{1}$, in $\mathscr{V}$, of the union af all points described in (i), (ii), (iï), with the following properties:

1) $\mathscr{G}_{1}$ is invariant under the complex Lorentz transformations $u_{j} \rightarrow \alpha u_{j}, v_{j} \rightarrow \frac{1}{\omega} v_{j}, \alpha \in \mathrm{C}-\{0\}$.

2) $\mathscr{G}_{1}$ is invariant under the reflection $u_{j} \leftrightarrow v_{j}$.

3) $\mathscr{G}_{1} \subset \mathscr{H}(\Delta) \cap \mathscr{V}$.

In order to obtain a domain in the space of the variables (II) [see (20), (21)], consider the following holomorphic mappings

$$
\left\{\begin{array}{l}
\mathscr{I}:\left(u_{1}, v_{1}, u_{2}, v_{2}\right) \in \mathscr{V}^{\prime} \rightarrow\left(z_{1}, z_{2}, z_{3}, \alpha\right) \in \mathbb{C}^{4} . \\
\mathscr{I}:\left(u_{1}, v_{1}, u_{2}, v_{2}\right) \in \mathscr{V} \rightarrow\left(z_{1}, z_{2}, z_{3}\right) \in \mathbb{C}^{3} .
\end{array}\right.
$$

It can be verified that the mapping $\mathscr{I}$ is open at all the points described in (i), (ii), (iii). (The only points where a careful verification is needed are those for which $\lambda\left(z_{1}, z_{2}, z_{3}\right)=0$, i. e. the vectors $\pi_{1}$ ans $\pi_{2}$ are colinear: there, the rank of the mapping is not maximal.) It follows that 
$\mathscr{I}\left(\mathscr{G}_{1}\right)$ is a neighborhood of $E$ (defined in (38)). Because of the invariance of $\mathscr{G}_{1}$, we have

$$
\mathscr{I}\left(\mathscr{G}_{1} \cap \mathscr{V}^{\prime}\right)=\mathscr{J}\left(\mathscr{G}_{1}\right) \times\{\mathbb{C}-\{0\}\}-\left\{z_{1} \leqq \mathrm{O}\right\} .
$$

We can state:

Lemma 1. The junctions $f_{1,2}\left(z_{1}, z_{2}, z_{3}, \alpha\right)$ are analytic in a domain $G_{1}$ of the following form:

$$
\begin{gathered}
G_{1}=\left\{\left(z_{1}, z_{2}, z_{3}, \alpha\right):\left|z_{1}-Z_{1}\right|+\left|z_{2}-Z_{2}\right|+\left|z_{3}-Z_{3}\right|<\varepsilon\left(Z_{1}, Z_{2}, Z_{3}\right),\right. \\
\left.\left(Z_{1}, Z_{2}, Z_{3}\right) \in E ; z_{1} \in \mathbb{R}^{-} \quad \alpha \neq 0\right\} .
\end{gathered}
$$

The domain $G_{1}$ can be obtained by removing the points $z_{1} \leqq 0$ from a neigborhood of the set

$$
\begin{gathered}
E_{1}=\left\{\left(z_{1}, z_{2}, z_{3}, \alpha\right): z_{1} \text { real }<\mathscr{M}_{1}^{2}, z_{2} \text { real } \leqq 0\right. \\
\left.\operatorname{Im} z_{3}>0 \quad \alpha \neq 0\right\}
\end{gathered}
$$

\section{b) Domain $G_{2}$}

We shall now describe a domain in the manifold $\mathscr{V}$ which is contained in the envelope of holomorphy $\mathscr{H} \mathscr{A} \mathscr{B}$ of the union of the tubes $\mathscr{A}$ and $8 \$$ and of a small open set $\mathscr{N} \Pi\left\{\operatorname{Im}\left(\pi_{1}+\pi_{2}\right) \in V^{+}\right\}$connecting $\mathscr{A}$ and $\mathscr{B}$ (see section 2). We recall that $\mathscr{N}$ is a complex neighborhood of the real region

$$
\left\{\pi_{1}^{2}<\mathscr{M}_{1}^{2} \pi_{2}^{2}<\mathscr{M}_{2}^{2}\left(\pi_{1}-\pi_{2}\right)^{2}<\mathscr{M}_{10}^{2}\right\} .
$$

For each $\sigma>0$, let $\mathscr{L}_{\sigma}$ be the complex submanifold of $\mathscr{V}$ defined by setting

$$
\left\{\begin{array}{l}
u^{+}=u_{1}+u_{2}=\sigma \\
v^{+}=v_{1}+v_{2}=\sigma
\end{array}\right.
$$

In $\mathscr{L}_{\sigma}$ one can take as variables the coordinates $u^{-}=u_{1}-u_{2}$ and $v^{-}=v_{1}-v_{2}$ of the vector $\pi_{1}-\pi_{2}$.

Let $\mathscr{D}_{\sigma}$ denote the Jost-Lehmann-Dyson domain defined in $\mathscr{L}_{\sigma}$ as the envelope of holomorphy ([13]) of the union of the tubes

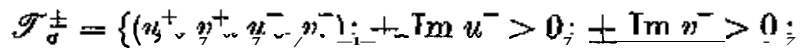

$$
\begin{aligned}
& \left.u^{+}-v^{+}=v\right\}
\end{aligned}
$$

with a neighborhood of the real region:

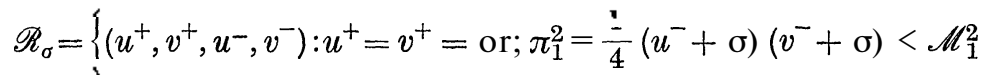

$$
\begin{aligned}
& \left.\pi_{2}^{2}=\frac{1}{\Lambda}(u-\sigma)(v-\sigma)<\mathscr{M}_{2}^{2} ;\left(\pi_{1}-\pi_{2}\right)^{2}=u \quad v<\mathscr{M}_{10}^{2}\right\} \text {. }
\end{aligned}
$$

$\mathscr{T}_{\sigma}^{+}\left(\right.$resp $\left.\mathscr{T}_{\sigma}^{-}\right)$is contained in the boundary of the tube $\mathscr{A}$ (resp. $\mathscr{B}$ ), and $\mathscr{R}_{\sigma}$ is the trace of the region (10) on the manifold $\mathscr{L}_{\sigma}$. Therefore $\mathscr{R}_{\sigma} \mathrm{C} \mathscr{N}_{\sigma}=\mathscr{N} \Pi \mathscr{L}_{\sigma}$. Every point in $\mathscr{L}_{\sigma}$ belonging to $\mathscr{T}_{\sim}^{+} \cup \mathscr{T}_{\sigma}^{-} U \mathscr{N}_{\sigma}$ 
has a neighborhood whose intersection with the tube $\left\{\operatorname{Im}\left(\pi_{1}+\pi_{2}\right) \in V^{+}\right\}$ is contained in $\mathscr{H}_{\mathscr{A} \mathscr{B}}$. On the other hand, every point $M-\left(\sigma, a, u_{0}^{-}, v_{0}^{-}\right)$ of $\mathscr{D}_{\sigma}$ belongs to the envelope of holomorphy of some relatively compact subdomain $K_{M}$ of $\mathscr{T}_{\sigma}^{+} \mathrm{U} \mathscr{T}_{\sigma}^{-} \mathrm{U} \mathscr{N}_{\sigma}$ (see, for instance, the process of analytic completion, using disks, given in [13]). One can therefore associate with $K_{M}$ a positive number $\rho\left(K_{M}\right)$ such that the domain

$$
\begin{gathered}
\left\{u^{+}, v^{+}, u^{-}, v^{-}:\left(\operatorname{cr}, \sigma, u^{-}, v^{-}\right) \in K_{M} ;\left|u^{+}-\sigma\right|+\left|v^{+}-\sigma\right|<\varrho\left(K_{M}\right)\right. \\
\left.\operatorname{Im} u^{+}>0, \operatorname{Im} v^{+}>0\right\}
\end{gathered}
$$

is contained in $\mathscr{H}_{\mathscr{A} \mathscr{B}}$. This domain being a topological product, so is its envelope of holomorphy. Therefore the following set of neighboring points of $M$ :

$$
\begin{array}{r}
\left\{\left(u^{+}, v^{+}, u^{-}, v^{-}\right) ;\left|u^{+}-\sigma\right|+\mid v^{+}-\sigma<\varrho\left(K_{M}\right)\right. \\
\left.u^{-}=u_{0}^{-}, v^{-}=v_{0}^{-} \operatorname{Im} u^{+}>0, \operatorname{Im} v^{+}>0\right\}
\end{array}
$$

is contained in $\mathscr{H}_{\mathscr{A} \mathscr{B}}$, hence in $\mathscr{H}(\Delta)$.

Thus it has been finall $\}^{\wedge}$ shown that $\mathscr{H}(\Delta)$ contains a domain of the form:

$$
\begin{gathered}
\underset{\sigma}{\mathrm{U}}\left\{\left(u^{+}, v^{+}, u^{-}, v^{-}\right):\left(u^{-}, v^{-}\right) \in \mathscr{D}_{\sigma} ;\left|u^{+}-\sigma\right|+\left|v^{+}-\sigma\right|<\rho\left(u^{-}, v^{-}\right)\right. \\
\left.\operatorname{Im} u^{+}>0, \operatorname{Im} v^{+}>0\right\} .
\end{gathered}
$$

Henceforth we shall restrict $\sigma$ to values such that

$$
\sigma>\mathscr{M}_{1}+\mathscr{M}_{2}
$$

In this case, it turns out that the inequalities $\pi_{1}^{2}<\mathscr{M}_{1}^{2}$ and $\pi_{2}^{2}<\mathscr{M}_{2}^{2}$ imply $\left(\pi_{1}-\pi_{2}\right)^{2}<\mathscr{M}_{10}^{2}$, so that, in the variables $u \sim$ and $v^{-}$, the region $\mathscr{R}_{\sigma}$ is bounded by the two intersecting branches of hyperbolae (see Fig. 5):

$$
\begin{aligned}
& \left(u^{-}+\sigma\right)(v \sim+\sigma)=4 \mathscr{M}_{1}^{2} ; u^{-}+\sigma>0 . \\
& \left(u^{-}-\sigma\right)\left(v^{-}-\sigma\right)=4 \mathscr{M}_{2}^{2} u^{-}-\sigma>0 .
\end{aligned}
$$

Since our purpose is to consider these domains in the neighborhood of the manifold $\mathscr{W}=\left\{\left(u^{+}, v^{+}, u^{-}, v^{-}\right): \pi_{1}^{2}-\pi_{2}^{2}=\mu_{1}^{2}-\mu_{2}^{2}\right\}$, we shall study, for every $\sigma>\mathscr{M}_{1}+\mathscr{M}_{2}$, the intersection of $\mathscr{D}_{\sigma}$ with the manifold $\mathscr{M}_{\sigma}=\mathscr{L}_{\sigma} \Pi \mathscr{W}$.According to (8), (16), (41), $\mathscr{M}_{\sigma}$ is defined in $\mathscr{L}_{\sigma}$ by the equation:

Define:

$$
a\left(u^{-}+v^{-}\right)=2\left(\mu_{1}^{2}-\mu_{2}^{2}\right) \text {. }
$$

$$
x=\frac{u^{-}-v^{-}}{4}
$$


In $\mathscr{M}_{\sigma}$ one can take as variables either $x$ or the Lorentz-invariant variable $z$ defined by (31): according to (35), (41), (48), (49), $z$ is related to $x$ by

$$
z=-x^{2}+\sigma^{2}-2\left(\mu_{1}^{2}+\mu_{2}^{2}\right)+\frac{\left(\mu_{1}^{2}-\mu_{2}^{2}\right)^{2}}{\sigma^{2}}-\Phi .
$$

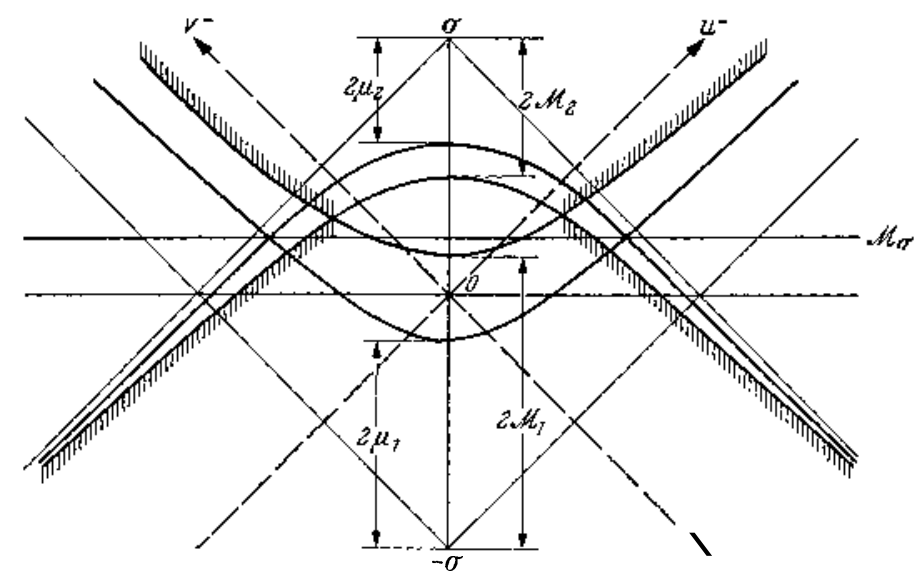

Fig. 5. Coincidence region for the Dyson domain in $\mathscr{L}_{\sigma}$

In order to simplify the calculations, we replace $\mathscr{D}_{\sigma}$ by a subdomain $\mathscr{D}_{\sigma}^{\prime}$ which is the Jost-Lehmann-Dyson domain corresponding to the (real) region $\mathscr{R}_{\sigma}^{\prime}$ defined by the inequalities:

$$
\left(u^{\prime-}+\sigma^{\prime}\right)\left(v^{\prime-}+\sigma^{\prime}\right)<4 \mathscr{K}^{2}
$$

and

where:

$$
\left(u^{\prime-}-\sigma^{\prime}\right)\left(v^{\prime-}-\sigma^{\prime}\right)<4 \mathscr{H}^{2}
$$

$$
\begin{aligned}
u^{\prime} & =u^{-}-\frac{\left(\mu_{1}^{2}-\mu_{2}^{2}\right)}{\sigma}, \\
V^{-} & =V^{-}-\frac{\left(\mu_{1}^{2}-\mu_{2}^{2}\right)}{\sigma}, \\
\sigma^{\prime} & =\sigma+\frac{\left|\mu_{1}^{2}-\mu_{2}^{2}\right|}{\sigma}, \\
\mathscr{H}^{2} & =\max \left(\mu_{1}^{2}+\frac{\Phi}{4}, \mu_{2}^{2}+\frac{\Phi}{4}\right) .
\end{aligned}
$$

It can be checked that $\mathscr{R}_{\sigma}^{\prime}$ is contained in $\mathscr{R}_{\sigma}$, so that $\mathscr{D}_{\sigma}^{\prime} \mathrm{C} \mathscr{D}_{\sigma}$. The advantage of $\mathscr{R}_{\sigma}^{\prime}$ is that it is symmetric with respect to the straight line $u^{\prime-}+v^{\prime-}=0$.

According to (48), (49), (53), (54), the manifold, $\mathscr{M}_{\sigma}$ is defined, in the new variables, by the equation:

$$
u^{\prime-}+v^{\prime-}=0 \text {. }
$$


One also has :

$$
x-\frac{\left(u^{\prime}--v^{\prime}\right)}{2} .
$$

By using the classical method $[9,12]$, it is easily seen that the "admissible hyperbolae" have the equation:

$$
\left(u^{\prime-}-\xi\right)\left(v^{\prime-}+\xi\right)-\eta^{2}=0
$$

where the point $(\xi, \eta)$ belongs to the following set $\Gamma$ (see Fig. 6)

with :

$$
\Gamma=\gamma^{+} \cup \gamma^{-} \cup \delta
$$

$$
\begin{aligned}
& \gamma^{ \pm}=\left\{(\xi, \eta) \in \mathbb{R}^{2}: \xi^{2}+(\eta \pm 2 \mathscr{M})^{2}-\sigma^{2} \leqq 0\right\} \\
& \delta=\left\{(\xi, \eta) \in \mathbb{R}^{2}: \xi^{2}-\sigma^{2} \leqq 0 ; \eta^{2}-4 \mathscr{M}^{2} \geqq 0\right\}
\end{aligned}
$$

The necessary and sufficient condition for a point of $\mathscr{M}_{\sigma}$ to be in $\mathscr{D}_{\sigma}^{\prime}$ is, according to (57), (58), (59), that:

that is:

$$
(x-\xi)^{2}+\eta^{2} \neq 0 \text { for all }(\xi, \eta) P E
$$

$$
x \neq \xi \pm \text { i } \eta \text { for all }(\xi, \eta) \in \Gamma \text {. }
$$

Thus, the intersection of $\mathscr{D}_{\sigma}^{\prime}$ and $\mathscr{M}_{\sigma}$ is represented, in the complex plane of the variable $x$ (which we can identify with the $(\xi, \eta)$ plane) by the complement of $\Gamma$ (note that $\Gamma$ is invariant under $\eta \rightarrow-\eta$ ).
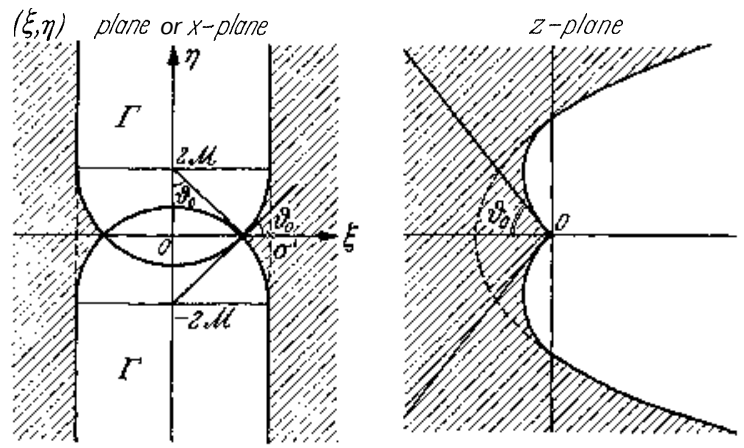

Fig. 6. The domain given by the Jost-Lehmann-Dyson domain in the $x$-plane (shaded) and its image in the $z$-plane

Let $\left.2 \theta 10<\theta<\frac{\pi}{2}\right)$ be the angle of the two circles bordering $\gamma^{+}$and $\gamma \sim$ (see Fig. 6). In the conformal mapping $x \rightarrow z$, (see (50)), this angle is conserved. It can be checked that the image of $\mathscr{D}_{\sigma}^{\prime} \cap \mathscr{M}_{\sigma}$ in the complex plane of $z$ contains the sector

$$
\{z:|\arg (-z)|<\theta\} \text {. }
$$

In particular, taking into account (55), (56), the formula (50) can be rewritten

$$
z=-x^{2}+\sigma^{2}-4 \mathscr{A}^{2}
$$


and the intersection points of the two circles bordering $\gamma^{+}$and $\gamma$ (see (60)) are mapped onto the origin in the $z$-plane. The value of $\theta$ is given by:

$$
\cos \theta=\frac{2 \varkappa}{\sigma^{\prime}} \leqq \frac{2 \varkappa}{\sigma} .
$$

Let $k$ be a real positive number such that

and define

$$
k>\max \left\{1, \frac{2 \cdot \mathscr{A}}{\mathscr{M}_{1}+\mathscr{K}_{2}}\right\} .
$$

$$
\sigma_{0}=k\left(\mathscr{H}_{1}+\mathscr{M}_{2}\right)>\mathscr{H}_{1}+\mathscr{H}_{2} \text {. }
$$

When $\sigma>\sigma_{0}, \theta$ is minorized by the angle $\theta_{0}$, independent of $\mathrm{cr}$, such that

$$
\cos \theta_{0}=\underset{k\left(\mathscr{M}_{1}+\mathscr{M}_{2}\right)}{2 \mathscr{M}} .
$$

We conclude that $\mathscr{H}(\Delta)$ contains a subdomain of the domain (44), which has the following form:

$$
\cup_{\sigma>\sigma_{0}}\left\{\begin{array}{l}
\left\{u^{+}, v^{+}, u^{-}, v^{-}\right):|\arg (-z)|<\theta_{0}, z=-\frac{\left(u^{-}-v^{-}\right)^{2}}{4}+\sigma^{2}-4 \mathscr{K}^{2} \\
\left|u^{+}-\sigma\right|+\left|v^{+}-\sigma\right|+\left|\frac{u^{-}+v^{-}}{2}-\frac{\mu_{1}^{2}-\mu_{2}^{2}}{\sigma}\right|<\rho(u-v, \sigma) \\
\operatorname{Im} u^{+}>0 \operatorname{Im} v^{+}>0 .
\end{array}\right.
$$

Taking the union of all transforms of this domain under the complex Lorentz transformations in $\mathscr{V}$, then removing the cut $\mathscr{C}$ (see (23)), we obtain a domain, the image of which, in the space of the variables (II) (see (20)), has the form:

$$
G_{2}=\cup_{\sigma}\left\{\begin{array}{l}
\left(z_{0}, z_{2}, z_{3}, \alpha\right):|\arg (-z)|<\theta_{0}, z=2\left(z_{1}+z_{2}-\mu_{1}^{2}-\mu_{2}^{2}\right)-\Phi \\
\left|z_{1}-z_{2}-\mu^{\hat{2}}+\mu_{2}^{2}\right|+\left|z_{3}-\sigma^{2}\right|<\varrho^{\prime}\left(z_{1}+z_{2}, \sigma\right) ; \\
\operatorname{Im} z_{3}>0 \quad z_{1} \notin \mathrm{R} \quad \alpha \neq 0 .
\end{array}\right.
$$

This can be stated as:

Lemma 2. The functions $f_{1,2}\left(z_{1}, z_{2}, z_{3}, \alpha\right)$ are analytic in a domain $G_{2}$ of the form (66), which is obtained by removing all points such that $z_{1} \leqq 0$ or $\operatorname{Im} z_{3} \leqq 0$ from some neighborhood of the set

$$
\begin{gathered}
E_{2}=\left\{\left(z_{1}, z_{2}, z_{3}, \alpha\right): z_{1}-z_{2}=\mu_{1}^{2}-\mu_{2}^{2} ; z_{3} \text { real }>\sigma_{0}^{2} ;\right. \\
\left.|\arg (-z)| \leqq \theta_{0} ; \alpha \neq 0\right\} .
\end{gathered}
$$

Exchanging the roles of the vectors $\pi_{1}+\pi_{2}$ and $\pi_{1}-\pi_{2}$, (in particular, replacing the tubes $\mathscr{A}, \mathscr{B}$ by $\mathscr{A}^{\prime}$ and $\mathscr{B}^{\prime}$ ), one obtains, by identical arguments a domain $G_{3}$, analogous to $G_{2}$, given by

$$
G_{3}=\bigcup_{\sigma>\sigma_{0}}\left\{\begin{array}{l}
\left(z_{1}, z_{2}, z_{3}, \alpha\right), * \arg (-z)_{N}<\theta_{v^{*}} \\
\left|z_{1}-z_{2}-\mu_{1}^{2}+\mu_{2}^{2}\right|+\left|2\left(z_{1}+z_{2}\right)-z_{3}-\sigma^{2}\right|<Q^{\prime \prime}\left(z_{1}+z_{2}, \sigma\right) ; \\
\operatorname{Im}\left[2\left(z_{1}+z_{2}\right)-z_{3}\right]<0 \quad z_{1} \notin \mathbb{R}^{-} \alpha 4=0 .
\end{array}\right.
$$


We can state:

Lemma 3. The functions $f_{1,2}\left(z_{1}, z_{2}, z_{3}, \alpha\right)$ are holomorphic in a domain $G_{3}$ of the form (67), which can be obtained by removing all points such that $z_{1} \leqq 0$ or $\operatorname{Im}\left[2\left(z_{1}+z_{2}\right) \sim z_{3}\right]=$ 0fromsomeneighborhood of the set

$$
\begin{aligned}
E_{3}= & \left\{\left(z_{1}, z_{2}, z_{3}, \alpha\right): z_{1}-z_{2}=\mu \backslash-\mu_{2}^{2}\right. \\
& \left.2\left(z_{1}+z_{2}\right)-z_{3} \text { real }>\sigma_{0}^{\lrcorner}|\arg (-z)|<\theta_{0} \alpha \neq 0\right\} .
\end{aligned}
$$

\section{5, Analytic completion in the invariants $s$ and $z$}

We shall now consider the intersections of the domains $G_{j}$ with the image $J\left(\mathscr{W}^{\prime}\right)$ of the manifold $\mathscr{W}^{\prime}(j=1,2,3)$ :

$$
\mathscr{I}\left(\mathscr{W}^{\prime}\right)=\left\{\left(z_{1}, z_{2}, z_{3}, \alpha\right): z_{1}-z_{2}=\mu_{1}^{2}-\mu_{2}^{2} z_{1} \notin \mathbb{R}^{-} \alpha \neq 0\right\} .
$$

The variables we shall use are $s$ (or $u$ ), $z, \alpha$ (see (34), (35)). The domains $G_{j} \Pi \mathscr{I}\left(\mathscr{W}^{\prime}\right)$ are topological products of certain domains in the space $\mathbb{C}^{2}$ of the variables $s, z$ by the fixed domain $\{\alpha \in \mathbb{C}-\{0\}\}$ :

$$
G_{j} \Pi \mathscr{I}\left(\mathscr{W}^{\prime}\right)=\left\{(s, z, \alpha)(s, z) \in G_{j}^{\prime} \quad \alpha \in \mathbb{C}-\{0\}\right\}=0 ; \times\{\mathbb{C}-\{0\}\} .
$$

Hence, to complete analytically $\left(G_{1} \cup G_{2} \cup G_{3}\right) \Pi \mathscr{I}\left(\mathscr{W}^{\prime}\right)$ is equivalent to analytically completing $G_{1}^{\prime} \cup G_{2}^{\prime} \cup G_{3}^{\prime}$, and then taking the topological product with $\{\mathbb{C}-\{0\}\}$.

\section{a) Completions of $G_{1}^{\prime} \mathrm{U} G_{2}^{\prime}$ and $G\left\{\mathrm{U} G_{3}^{\prime}\right.$}

When $\mu_{1}^{2}-\mu_{2}^{2} \geqq 0$, according to Lemma 1 (or formula (40)), $G_{1}^{\prime}$ is of the form

$$
\mathscr{N}\left(E_{1}^{\prime}\right) \cap\left\{z_{1}=z_{2}+\mu_{1}^{2}-\mu_{2}^{2} \notin \mathbb{R}^{-}\right\}
$$

where $\mathscr{N}\left(E_{1}^{\prime}\right)$ is an open neighborhood of the set

$$
E_{1}^{\prime}=\left\{\left(z_{2}, z_{3}\right): z_{2} \text { real } \leqq 0 \quad \operatorname{Im} z_{3}>0\right\}
$$

When $\mu_{1}^{2}-\mu_{2}^{2} \geqq 0$, one obtains the analogous domain

$$
\mathscr{N}\left(E_{1}^{\prime \prime}\right) \cap\left\{z_{1}=z_{2}+u_{1}^{2}-\mu_{2}^{2} \notin \mathbb{R}^{-}\right\}
$$

$\mathscr{N}\left(E_{1}^{\prime \prime}\right)$ is an open neighborhood of

$$
E_{1}^{\prime \prime}=\left\{\left(z_{2}, z_{3}\right): z_{1} \text { real } \leqq 0 \quad \operatorname{Im} z_{3}>0\right\} .
$$

It may be noticed that the symmetry in $z_{1}$ and $z_{2}$ is not complete because of the unsymmetrical change of variables (I) $\rightarrow$ (II). In the variables $s$ and $\mathrm{z}$, we find the following expression, valid for both signs of $\mu_{1}^{2}-\mu_{2}^{2}$ :

$$
0 ;=\mathscr{N}\left(\mathscr{E}_{1}\right) \cap\left\{z^{+} 4 \mu_{1}^{2+} \Phi \$ \mathbb{R}^{-}\right\}
$$

where $\mathscr{N}\left(\mathscr{E}_{1}\right)$ is a neighborhood of

$\alpha(t)$ is given by

$$
\mathscr{E}_{1}=\{(s, z): \text { z real } \leqq-a(t) \quad \operatorname{Im} s>0\}
$$

$$
a(t)=\min \left(4 \mu_{1}^{2}+\Phi, 4 \mu_{2}^{2}+\Phi\right) .
$$


On the other hand, using (66) and (67) (or lemmas 2 and 3), we find

$$
\begin{aligned}
& \left.G_{2}^{\prime}=\mathscr{N} \mathscr{E}_{2}\right) \cap\{\operatorname{Im} s>0\} \Pi\left\{z+4 \mu_{1}^{2}+\Phi \notin \mathbb{R}^{-}\right\}, \\
& G_{3}^{\prime}=\mathscr{N}\left(\mathscr{E}_{3}\right) \cap\{\operatorname{Im} u=\operatorname{Im}(z-s)<0\} \Pi\left\{z+4 \mu_{1}^{2}+\Phi \notin \mathbb{R}^{-}\right\},
\end{aligned}
$$

where $\mathscr{N}\left(\mathscr{E}_{2}\right)$ and $\mathscr{N}\left(\mathscr{E}_{3}\right)$ are respectively open neighborhoods of

with :

$$
\begin{aligned}
& \mathscr{E}_{2}=\left\{(s, z):|\arg (-z)|<\theta_{0} ; s>s_{0}(t)\right\} \\
& \mathscr{E}_{3}=\left\{(s, z):|\arg (-z)|<\theta_{0} ; \mathrm{u}>u_{0}(t)\right\},
\end{aligned}
$$

$$
\begin{aligned}
& s_{0}(t)=\sigma_{0}^{2}+\frac{1}{4 t}\left(m_{1}^{2}-\mathbf{m i}+m_{0}^{2}-m_{2}^{2}\right)^{2}, \\
& u_{0}\left(\mathrm{O}=\sigma_{0}^{2}+\frac{1}{4 t}\left(m_{1}^{2}-m_{3}^{2}+m_{2}^{2}-m_{0}^{2}\right)^{2} .\right.
\end{aligned}
$$

In order to find an analytic completion of $G_{1}^{\prime} U G_{2}^{\prime}$, we make the following change of variables:

$$
s^{\prime}=\log \left(s-s_{0}\right) ; z^{\prime}=\arg \operatorname{ch}\left[1-2\left(-\frac{z}{a}\right)^{\pi / \theta_{0}}\right],
$$

which transforms $\left(G_{1}^{\prime} \mathrm{U} G_{2}^{\prime}\right) \Pi\left\{z+a(t) \notin \mathbb{R}^{-}\right\}$into a domain of the type

$$
\mathscr{N}^{\prime}\left(E^{\prime} \Pi\left\{\left(s^{\prime}, z^{\prime}\right) ; 0<\operatorname{Im} s^{\prime}<\pi ; 0<\operatorname{Im} z^{\prime}<\pi\right\}\right.
$$

where $\mathscr{N}^{\prime}\left(E^{\prime}\right)$ is a neighborhood of the "flattened" tube:

$$
\begin{gathered}
E^{\prime}=\left\{\left(s^{\prime}, z^{\prime}\right): \operatorname{Im} s^{\prime}=0 \quad 0 \leqq \operatorname{Im} z^{\prime} \leqq \pi\right\} \mathrm{U} \\
\mathrm{U}\left\{\left(s^{\prime}, z^{\prime}\right) ; 0 \leqq \operatorname{Im} s^{\prime} \leqq \pi \operatorname{Im} z^{\prime}=\pi\right\} .
\end{gathered}
$$

A refined version of the tube theorem shows that the envelope of holomorphy of this domain contains the convex envelope of the "flattened tube," namely:

$$
\left\{\left(s^{\prime}, z^{\prime}\right): 0<\operatorname{Im} s^{\prime}<\pi 0<\operatorname{Im} z^{\prime}<\pi \operatorname{Im} z^{\prime}>\operatorname{Im} s^{\prime}\right\}
$$

which one can also write as a union of topological products:

$$
\bigcup_{0<\beta<\pi}\left\{\left(s^{\prime}, z^{\prime}\right)_{j}<\operatorname{Im} s^{\prime}<; .<\operatorname{Im} z^{\prime}<\pi .\right.
$$

The value of $z$ corresponding to the mass-shell is $z=-\Phi$, to which corresponds (see (71)) the value $z^{\prime}-i \varphi$ such that

$$
\cos \varphi=1-2\left(\frac{Q-1}{(\omega)}\right)^{\pi / \theta_{0}} .
$$

From (72), we extract a topological product corresponding to a value $\beta=\psi$ such that $0<\psi<\varphi<\pi$. Its inverse image in the variables $(s, z)$ is a topological product

$$
\left\{(s, \mathrm{z}) \cdots 0<\arg \left(s-s_{0}\right)<\psi ; z\{A\}\right.
$$

where $A$ is a domain containing the "physical point" $z--\Phi$. 
The boundary of $A$ is a curve with the following properties (see Fig.7): it is convex, symmetric with respect to the real axis and has the asymptotic directions:

where:

$$
\arg z= \pm(\pi-\chi),
$$

$$
\chi=\theta_{0}\left(1-\frac{\psi}{\pi}\right) \text {. }
$$

It passes through the real point $z=-\Psi$, defined, according to (71), by

$$
\Psi=a\left(\sin -\frac{\psi}{2}\right)^{2 \theta_{0} / \pi}
$$

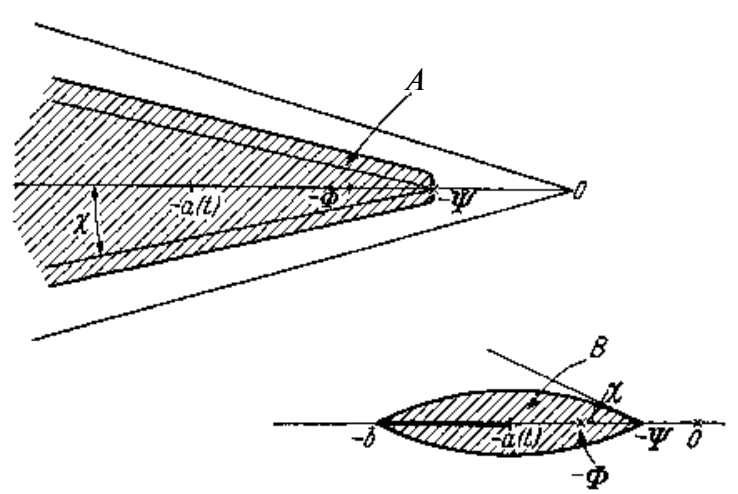

Fig. 7. The domains $A$ and $B$ in the $z$-plane

One can then replace $A$ by a subdomain $B$ of the following form (see Fig. 7):

$$
B=\left\{\begin{array}{l}
\mathfrak{1} \\
1
\end{array} \pi-\chi<\left|\arg \frac{z+\Psi}{z+b}\right| \leqq \pi, z+a(t) \notin \mathbf{R}^{-}\right\}
$$

where $b$ is real and satisfies the condition: $b>a(t)$. The boundary of $B$ is composed of two arcs of circles with extremities $-\Psi$ and $-b$, and of the real segment $[-b,-a(t)]$. It is clear that $B$ still contains the physical point $z=-\Phi$. We can state:

Lemma 4. The functions $f_{1,2}\left(z_{1}, z_{2}, z_{3},\left.\alpha\right|_{\mathscr{I}(\mathscr{y})}\right.$ are holomorphic in the domain $\Delta_{\mathbf{2}} \mathrm{X}\{\alpha \neq 0\}$ where

$$
\Delta_{2}=\left\{(s, z): 0<\arg \left(s-s_{0}\right)<\psi 2 \in B\right\} .
$$

By applying the same process of completion to $G_{1}^{\prime} U G_{3}^{\prime}$, we obtain the analogous result:

Lemma 5. The functions $\left.f_{1,2}\left(z_{1}, z_{2}, z_{3}, \alpha\right)\right|_{\mathscr{I}\left(\mathscr{W}^{\prime}\right)}$ are holomorphic in the domain $\Delta_{3} \times\{\alpha \neq 0\}$, where

$$
\Delta_{3}=\left\{(s, z) ;-\psi<\arg \left(u-u_{0}\right)<0 ; z \in B\right\} .
$$




\section{b) Construction of the "crossing domain"}

For reasons which shall be explained later, we shall not attempt to find the best possible domain, but only to prove that there exists a "crossing domain." We shall therefore use only subdomains of $G_{1}^{\prime}, \Delta_{2}, \Delta_{3}$, chosen so as to simplify the computations as much as possible. In particular we shall treat unsymmetrically the variables $s$ and $u$ (related by (33)), and use only domains which are topological products in $s$ and $z$. In order to extract from $\Delta_{3}$ such a topological product, we take, in the $s$-plane, the intersection of all sectors

$$
\left\{s ;-\psi<\arg \left(u-u_{0}\right)=\underset{s-p / o n e}{\left.\arg \left(-s-t+z+\Sigma m_{j}^{2}+\Phi-u_{0}\right)<0\right\}}\right.
$$

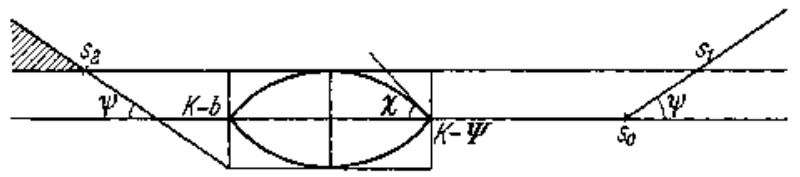

Fig. 8. $\Delta_{2}^{\prime j}$ is the topological product of $B$ (in the $z$-plane) by the shaded domain in the $s$-plane.

$$
K=-u_{0}-t+\sum_{j} m_{j}^{2}+\Phi
$$

for all values of $z$ in $B$. It is clear (see Fig. 8) that this intersection contains the domain

where

$$
\Delta_{2}^{\prime}=\left\{(s, z): \pi-\psi<\arg \left(s-s_{2}\right)<\pi ; z \in B\right\}
$$

$$
s_{\mathrm{2}}=\Phi+\sum_{j=0}^{3} m_{j}^{2}-u_{0}-t-b+\frac{b--\psi}{2} \operatorname{tg} \frac{\chi}{2}\left(i-\frac{2}{\operatorname{tg} \psi}\right) \text {. }
$$

Let $s_{1}$ be the intersection of the two straight lines $\left\{\operatorname{Im} s-\operatorname{Im} s_{2}\right\}$ and $\arg \left(s-s_{0}\right)=\psi$

$$
s_{1}=s_{0}+\frac{(b-\Psi) \operatorname{tg} \frac{\chi}{2}}{2 \operatorname{tg} \psi}+i \frac{b-\psi}{2} \operatorname{tg} \frac{Z}{2} .
$$

We finally extract from $G_{1}^{\prime}$ and $\Delta_{2} \mathrm{U} \Delta_{2}^{\prime}$ the following open sets :

$$
\begin{gathered}
D_{1}=G_{1}^{\prime} \Pi\left\{s: \operatorname{Im} s>\operatorname{Im} s_{2}\right\} \\
D_{2}=\left\{(s, z): z \in B ; \operatorname{Im} s>\operatorname{Im} s_{2} ; s \in \mathscr{N}\left(\left\{s=s_{1}+\rho, \rho>0\right\}\right)\right. \\
\text { or } \left.s \in \mathscr{N}\left(\left\{s=s_{2}-\varrho^{\prime}, \varrho^{\prime}>0\right\}\right)\right\} .
\end{gathered}
$$

Here $\mathscr{N}\left(\left\{s=s_{1}+\rho, \rho>0\right\}\right)$, and $\mathscr{N}\left(\left\{s=s_{2}-\varrho^{\prime}, \varrho^{\prime}>0\right\}\right)$ denote open neighborhoods of the corresponding sets.

We apply to these domains the conf ormal mappings

$$
\begin{aligned}
& s^{\prime \prime}=s-\frac{i}{2}(b-\Psi) \operatorname{tg} \frac{x}{2} \\
& z^{\prime \prime}=\arg \operatorname{ch}\left\{1-2\left[-\frac{(z+\Psi)(b-a)}{(a-\bar{\Psi})(b-z)}\right]^{\pi / x}\right\}
\end{aligned}
$$


and denote

$$
s_{1}^{\prime \prime}=\operatorname{Re} s_{1}, s_{2}^{\prime \prime}-\operatorname{Re} s_{2}
$$

The image of $B$ in the complex plane of $z^{\prime \prime}$ is the strip $0<\operatorname{Im} z^{\prime \prime}{ }^{\prime}<\pi$; the cut $-b \leqq 25 \leqq-$ a has been unfolded and mapped onto the line $\left\{\operatorname{Im} z^{\prime \prime}=\pi\right\}$; the remainder of the boundary of $B$ is mapped onto the axis $\left\{\operatorname{Im} z^{\prime \prime}=0\right\}$.

The images of $D_{1}$ and $D_{2}$ in the variables $\left(\mathrm{s}^{\prime \prime}, z^{\prime \prime}\right)$ are thus:

$$
\begin{aligned}
D_{1}^{\prime \prime}= & V_{1}^{\prime \prime} \mathrm{n}\left\{\operatorname{Im} s^{\prime \prime}>0, \operatorname{Im} z^{\prime \prime}<\pi\right\} . \\
D_{2}^{\prime \prime}= & \left\{\left(s^{\prime \prime}, z^{\prime \prime}\right): 0<\operatorname{Im} z^{\prime \prime}<\pi s^{\prime \prime} \in \mathscr{N}\left(\left\{s^{\prime \prime}=s_{1}^{\prime \prime}+\rho, \rho>0\right\}\right)\right. \\
& \text { or } \left.5 " \in \mathscr{N}\left(\left\{s^{\prime \prime}=s_{2}-\varrho^{\prime}, \varrho^{\prime}>0\right\}\right) \operatorname{Im} s^{\prime \prime}>0\right\} .
\end{aligned}
$$

Here $\mathscr{N}\left(\left\{s^{\prime \prime}=s^{\prime \prime}+\varrho, \rho>0\right\}\right)$, if $\left(\left\{s^{\prime \prime}=s_{2}^{\prime \prime}-\varrho^{\prime}, \varrho^{\prime}>0\right\}\right)$ are open neighborhoods of the corresponding sets. $V^{\prime \prime}$ is an open neighborhood of the set:

$$
\left\{\left(s^{\prime \prime}, \mathrm{z}\right): \operatorname{Im} z^{\prime \prime}=\pi \quad \operatorname{Im} s^{\prime \prime} \geqq 0\right\} .
$$

Thus $D_{1}^{\prime \prime} \mathrm{U} D_{2}^{\prime \prime}$ contains the intersection of $\left\{\operatorname{Im} z^{\prime \prime}<\pi, \operatorname{Im} s^{\prime \prime}>0\right\}$ with an open neighborhood of the "flattened" semi-tube

$$
\begin{aligned}
\left\{\left(s^{\prime \prime}, z^{\prime \prime}\right): \operatorname{Im} z^{\prime \prime}\right. & \left.=\pi \operatorname{Im} s^{\prime \prime} \geqq 0\right\} \text { U }\left\{\left(s^{\prime \prime}, z^{\prime \prime}\right): 0<\operatorname{Im} z^{\prime \prime}<\pi,\right. \\
s^{\prime \prime} & \left.=s_{1}^{\prime \prime}+\varrho, \varrho>0 \text { or } s^{\prime \prime}=s_{2}^{\prime \prime}-\varrho^{\prime}, \varrho^{\prime}>0\right\} .
\end{aligned}
$$

A refined version of BREMERMANN's semi-tube theorem [15] asserts that the envelope of holomorphy of $D_{1}^{\prime \prime} \mathrm{U} D_{2}^{\prime \prime}$ contains the domain obtained by constructing the envelope of holomorphy of the "flattened" semitube in the same way as in the case of an open semi-tube. The solution of this problem is an open domain, the boundary of which is given by the harmonic function

$$
\operatorname{Im} z^{\prime \prime}=\operatorname{Im} \log \frac{s^{\prime \prime}-s_{1}^{\prime \prime}}{s^{\prime \prime}-s_{\circ}^{\prime \prime}}=\arg \left(s^{\prime \prime}-s_{1}^{\prime \prime}\right) \arg \left(s^{\prime \prime}-s_{2}^{\prime \prime}\right),
$$

and the domain is defined by:

$$
\begin{gathered}
\left\{\left(s^{\prime \prime}, z^{\prime \prime}\right): \operatorname{Im} s^{\prime \prime}>0 ; 0<\operatorname{Im} z^{\prime \prime}<\pi ; \operatorname{Im} z^{\prime \prime}>\arg \left(s^{\prime \prime}-s_{1}^{\prime \prime}\right)-\arg \left(s^{\prime \prime}-s_{2}^{\prime \prime}\right),\right. \\
\text { with } \left.0<\arg \left(s^{\prime \prime}-s^{\prime \prime}\right)<\pi, 0<\arg \left(s^{\prime \prime}-s_{2}^{\prime \prime}\right)<\pi\right\} .
\end{gathered}
$$

Consider the physical value $z=-\Phi$, to which corresponds

$$
\begin{aligned}
z^{\prime \prime} & =\arg \operatorname{ch}\left\{1-2\left[\frac{(\Phi-\Psi)(b-a)}{(a-\Psi)(b-\Psi)}\right]^{\pi / x}\right\}= \\
& =i \operatorname{Arccos}\left\{1-2\left[\frac{(\Phi-\Psi)(b-a)}{(a-\Psi)(b-\Psi)}\right]^{\pi / x}\right\}=i \varphi^{\prime \prime} .
\end{aligned}
$$

The intersection of the domain (78) with $\left\{z^{\prime \prime}=i \varphi^{\prime \prime}\right\}$ is given, in the upper half $s^{\prime \prime}$-plane, by the exterior of a (very large) circle passing through $s_{1}^{\prime \prime}$, and $s_{2}^{\prime \prime}$, defined by

$$
\arg \left(s^{\prime \prime}-s_{1}^{\prime \prime}\right)-\arg \left(s^{\prime \prime}-s_{2}^{\prime \prime}\right)<\varphi^{\prime \prime}, \operatorname{Im} s^{\prime \prime}>0 \text {. }
$$


Going back to the variables $s$ and $z$, one finds that for $z=-\boldsymbol{\Phi}$, i. e. on the physical mass-shell, the domain of analyticity of the functions $f_{1,2}\left(z_{1}, z_{2}, z_{3}, \alpha\right)$ contains the region $D \times\{\alpha \Phi 0\}$, with:

$$
\begin{aligned}
D=D(t) & =\left\{s: \arg \left(s-s_{0}\right)<\psi\right\} \cup \\
& \mathrm{U}\left\{s: \pi-\psi<\arg \left(s+t+u_{0}-\Sigma m_{j}^{2}\right)<\pi\right\} \mathrm{U} \\
& \cup\left\{s: \operatorname{Im} s>\frac{b-\boldsymbol{\psi}}{2} \operatorname{tg} \frac{\chi}{2}\right. \\
& \left.0<\arg \left(s-s_{1}\right)-\arg \left(s-s_{2}\right)<\varphi^{\prime \prime}\right\} .
\end{aligned}
$$

(see Fig. 9).

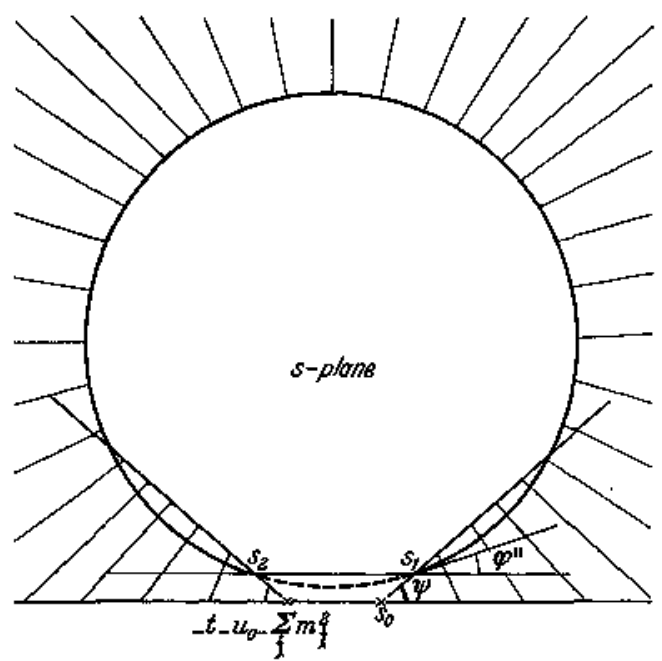

Fig. 9. The crossing Domain $\boldsymbol{D}(\boldsymbol{t})$ (shaded)

We note that, due to (64), (69), (70), $s=s_{0}$ and $s=-t-u_{0}+\Sigma m f$ define points in the physical regions $S$ and $U$, respectively so that $D$ provides the connection between $S^{+}$and $U^{-}$which is the object of this study*. To cast the result in the form announced in section 1 , we notice that, according to the remarks in sections 2 and 3 the points $\left(s, t, u, \zeta_{j}\right)$

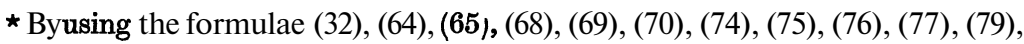
the parameters $s_{0}, u_{0}, s_{1}, s_{2}, \chi, \Psi, \varphi^{\prime \prime}$ which appear in the description of the set $D(t)$ can be reexpressed in terms of $t$, of the various masses $m_{i}, M_{i}$, and of the three independent parameters $k, b, \psi, k$ has to be chosen according to the formula (63) $\psi$ and $\delta$ have to satisfy the relations:

$$
\begin{aligned}
& 0<\psi<\varphi(t), \\
& b>a(t) .
\end{aligned}
$$

If we were interested in computing the largest domain possible by this method, we should take the union of the domains $D(t)$ for all possible values of $k, b, \psi$. 
such that $t<0, \zeta_{j}=m_{j}^{2}, s \in D=D(t)$ are in the image $/(\mathscr{H}(\Delta))$ of $\mathscr{H}(\Delta)$ in the variables $s, t, u, \zeta_{j}$. Since $D(t)$ does not intersect the trace of the manifold $\left\{Q\left(s, t, u, \zeta_{j}\right)=0\right\}$ on the mass shell, these points are in $I(\mathscr{H}(\Delta)$ $\cap\{Q(I(k)) \neq 0\})$. The mapping / is open on $t f(\Delta) \cap\{Q(I(k)) \neq 0\}$, which is $I$-saturated (see [5] and section 1). It follows that:

Theorem. For every real negative $t_{1}$ there exists a certain domain $\mathscr{W}_{t}^{+}\left(t_{1}\right)$ in the space of the variables $s, t, u$, and $\zeta_{j}$ (related by (4)) such that

1) $\mathscr{W}_{t}^{-}\left(t_{1}\right)$ is an open neighborhood of

$$
\left\{\left(s, t, u, \zeta_{j}\right): t=t_{i} ; \zeta_{j}=m_{j}^{2} ; s \in D\left(t_{1}\right)\right\}
$$

in particular it contains a set of the form:

$$
\begin{aligned}
& \left.\Omega_{t}^{+}\left(t_{1}\right)=\left\{\left(s, t, u, \zeta_{j}\right): \zeta_{j}=m_{j}^{2} ; \operatorname{Im} s>0|s|>5 \mathrm{ft}\right) ;\left|t-t_{1}\right|<\varepsilon\left(t_{1}, s\right)\right\} . \\
& \text { 2) } \left.I^{-1} \mathscr{W}_{i}^{+}\left(t_{1}\right)\right) \subset \mathscr{H}(\Delta) .
\end{aligned}
$$

By obvious permutations one proves the existence of $\Omega_{t}^{-}\left(t_{1}\right), \Omega_{s}^{ \pm}\left(i_{1}\right)$, $\Omega_{u}^{\ddagger}\left(u_{1}\right)$ announced in the introduction. The proof is now completed.

\section{Conclusion}

Several remarks can be made about this result.

a) In all cases when dispersion relations have been proved in quantum field theory, the crossing property between the corresponding two channels has ipso facto been demonstrated*. The new feature of the present approach is that it is valid in all mass cases, and for all negative momentum transfers.

b) We have already called the reader's attention to the fact that the proof does not use the Steinmann relations, whereas these relations play an essential (although implicit) role in the classical derivations of dispersion relations $[7-11]$. If one uses the Steinmann relations together with the methods indicated in this paper, one can take advantage, as in the classical proofs just mentioned, of the analyticity of the "absorptive part." This leads to a much larger domain of analyticity. However, because it requires somewhat lengthy distribution-theoretical arguments, we reserve this subject for a later article. For this reason, we did not attempt to compute the largest possible size for the domain obtained here; this domain must only be considered as supplying a general existence proof for a "crossing" domain of analyticity on the mass-shell.

* Strictly speaking, these proofs give only the "crossing" in the sense of analyticity in one variable only (for instance in $s$, for fixed $t$ ). However the corresponding points are actually points of analyticity in two variables. This follows from the work of H. LeHManN [16], and can also be seen by the methods used in the present paper. 
c) No properties of Lorentz covariance have been imposed on the function $\boldsymbol{H}(k)$. This makes the result valid also in the framework of $\boldsymbol{H}$. ARAKI and R. HAAG (see [17]).

d) Using the assumption that the Steinmann functions are tempered, it is possible to prove that $\boldsymbol{H}(k)$ has at most polynomial growth at infinity in the domain which has been derived in this paper. This proof will be given elsewhere. This fact, as remarked by A. MARTin, allows generalizations of "Pomeranchuk type" theorems to cases where dispersion relations have not been proved.

Acknowledgments. The authors are indebted for helpful discussions to Drs. R. Stora, A. Martin, H. Borchers, D. Ruglle. The second-named author wishes to thank Dr. L. Motchane for his kind hospitality at the "Institut des Hautes Etudes Scientifiques" in Bures.

\section{References}

[1] Lemmann, H., K. Symanzik, and W. Zimmermann: Nuovo Cimento 1, 205 (1954); 6, 319 (1957); Glaser, V., H. LeHMANN, and W. ZimmermanN: Nuovo Cimento 6, 1122 (1957).

[2] BROS, J., H. EPSTEIN, and V. GLASER: Nuovo Cimento 31, 1265 (1964).

[3] STREATER, R. F.: Journ. Math. Phys. 3, 256 (1962).

[4] BROS, J.: Séminaire Lelong (1962) $n^{\circ} 8$ (Paris 1962).

[5] HALL, D., and A. S. Wrghtman: Mat. Fys. Medd. Dan. Vid. Selsk. 31, n 5 (1957).

[6] HEPP, K.: Helv. Phys. Acta 36, 355 (1963).

[7] Bogoutubov, N. N., B. V. Medvedev, and M. K. Polivanov: Voprossy Teorii Dispersionnykh Sootnoshenii (Moscow 1958).

[8] Bremermann, H. J., R. Omhme, and J. G. TAYlOR: Phys. Rev. 109, 2178 (1958).

[9] LEHMANN, H.: Suppl. Nuovo Cimento 14, 153 (1959).

[10] Omniss, R.: In: Relations de Dispersion et Particules élémentaires. Paris: Hermann 1960.

[11] FROISSART, M.: In: Dispersion Relations and their Connection with Causality. New York: Academic Press 1964.

[12] Dyson, F. J.: Phys. Rev. 110, 1460 (1958).

[13] BROS, J;; A. Messiat, and R. STQRA: J. Math. Phys. 2, 639 (1961).

[14] Laray, J.: Bull, Soc. Math. France 85, 414 (1957).

[15] Bremerimann: H. J.: Math. Ann. 127, 406 (1954).

[16] Lemmann, H.: Lecture at the International School of Physics "Enrico Fermi". Varenna, Italy 1963.

[17] ARAKI, H.: Einführung in die Axiomatische Quantenfeldtheorie. E.T.H. Lecture Notes. Zurich 1962. 